\title{
Zermelo Navigation Problem in Geometry
}

\author{
Zdeněk Dušek \\ Institute of Technology and Business in České Budějovice \\ Faculty of Technology \\ Department of Informatics and Natural Sciences \\ Czech Republic \\ e-mail: zdusek@mail.vstecb.cz
}

\begin{abstract}
DOI 10.17818/NM/2018/4SI.16
UDK 514:656.61

Preliminary communication

Paper accepted: 28. 8. 2018.
\end{abstract}

\begin{abstract}
Summary
One of the long standing problems in navigation is explained and the mathematical formulation using Riemannian and Finsler geometry is introduced. Randers norms and Randers metrics used for the description of the influence of the wind or of the current on the cruising boat are explained. The explicit formulas relating the vector field representing the wind or the current with the corresponding Randers metric are given. Special situations with complete mathematical solution of the problem are mentioned, namely the case of the Riemannian manifold with constant sectional curvature, Finsler spaces with constant flag curvature, some special manifolds in dimension 3 and other. Some possibilities for different mathematical approach to the problem and also for further research are indicated.
\end{abstract}

\section{KEY WORDS}

Riemannian manifold

Finsler space

Geodesic

Zermelo navigation problem

\section{INTRODUCTION}

It is well known that the shortest path in the Euclidean space $E^{n}$ with the standard norm, which induces the distance function, is the straight line. On the sphere $S^{n}$, again with the natural distance function induced by the norm defined on tangent vectors and inherited from the standard norm on the ambient space $E^{n+1}$, locally, the shortest path is a part of the main circle. On a general Riemannian manifold, this is the property of geodesic curves. However, in a more general context, the norm does not have to come from the Euclidean scalar product. In the more general setting, a Minkowski norm on a vector space is not symmetric, hence the length of a vector may be different than the length of the opposite vector. Consequently, in a Finsler space, the length of a curve travelled in one direction may be different than the length of the same trajectory travelled in the opposite direction. However, geodesics are still well defined as shortest curves connecting their points that are close enough.

Imagine a ship sailing in a sea without any current and without any wind. The situation can be well described by the tools of Riemannian geometry, where shortest curves (fastest trajectories) are geodesics. However, if a wind or a current is present, then obviously the time required to travel some distance in one direction is different than the time necessary to travel the same distance in the opposite direction. There are various geometrical techniques how to describe this situation. One of them is the mentioned Finsler geometry, where the wind or the current modify the Riemannian metric to a general Finsler metric. We shall introduce this description and some interesting related phenomena.

\section{ELEMENTARY RIEMANNIAN AND FINSLER GEOMETRY}

Let us recall that the Euclidean vector space is a vector space with a symmetric and positively definite bilinear form . This bilinear form induces the norm (length) of vectors by the formula

$$
|v|=\sqrt{g(v, v)} \text {. }
$$

Given a Riemannian manifold $(M, g)$ we have a bilinear form $g_{\mathrm{x}}$ on the tangent space of each point $x$ of $M$. For example, let $(M, g)$ be the two-dimensional sphere in the three-dimensional space $E^{3}$. The tangent space at any point $x$ of $M$ is the tangent plane, as we naturally imagine it. Another example of a twodimensional manifold is the torus.
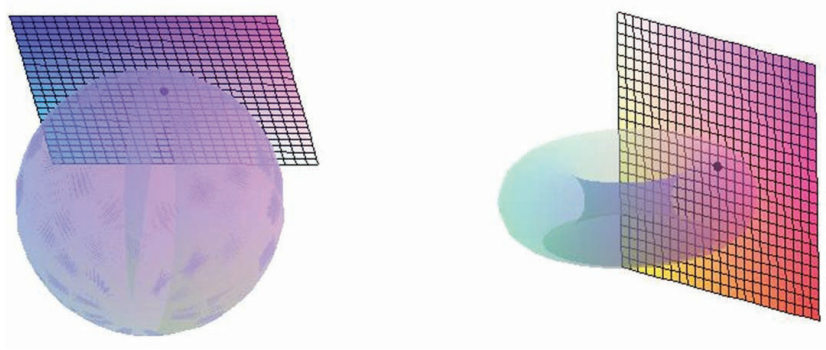

Source: author

Figure 1 The tangent plane of the sphere and the tangent plane of the torus at a point

The bilinear form $g_{x}$ is the restriction of the ordinary scalar product defined on the Euclidean space $E^{3}$ to this tangent plane. The induced lengths of vectors in these tangent planes are, in this situation, also the ordinary lengths as we naturally imagine it.

Now, let us consider a curve $\gamma(t)$ in the Riemannian manifold defined on the interval $(a, b)$. At each point $x$ on the curve, there is the bilinear form $g_{x}$ on the tangent space of the manifold $M$ at $x$. This bilinear form gives the length of the tangent vector $\gamma^{\prime}(t)$ at the point $\gamma(t)$. We naturally define the length of the curve as the integral of lengths of tangent vectors along this curve, hence

$$
L(\gamma)=\int_{a}^{b}\left|\gamma^{\prime}(t)\right| d t
$$

This integral does not depend on the parametrization of the curve. If we reparametrize the curve in a way that the norm of the tangent vector is equal to one, then $L(\gamma)=b-a$ and the 
length of the curve is the time necessary for traveling along the curve with the unit speed. Using the length of curves, we can define the distance of two points $x$ and $y$ of $M$ as the infimum of lengths of all curves connecting and $y$. For any two points $x$ and $y$ in a Riemannian manifold, the distance from $x$ to $y$ is the same as the distance from $y$ to $x$.

Geodesics in the Riemannian manifold $(M, g)$ are curves which are extremals of the length functional and they are the best possible analogues of lines in the Euclidean space. The important property of a geodesic $\gamma$ is that for any two points on $\gamma$ which are close enough, $\gamma$ is the shortest curve connecting these two points. Hence, the distance of these two points is realized by this geodesic $\gamma$. Geodesics are obtained as solutions of a system of differential equations. Another important property is that, given a point $x$ in $M$ and a tangent vector $v$ in the tangent space of $M$ at $x$, there exist a unique local geodesic $\gamma(t)$ such that $\gamma(0)=x$ and $\gamma^{\prime}(0)=v$ in other words, the position at the beginning is $x$ and the velocity at the beginning is $v$. If the Riemannian manifold $(M, g)$ is complete, then for any $x$ of $M$ there is a neighbourhood $U$ of $x$ such that any point $y$ of $U$ can be joined with $x$ by the unique minimizing geodesic lying in $U$. In the Euclidean space with the standard norm, geodesics are the straight lines. In the sphere with the natural Riemannian metric, geodesics are the main circles. The above mentioned properties of geodesics allow us to consider geodesics as the most natural trajectories in general Riemannian manifolds.

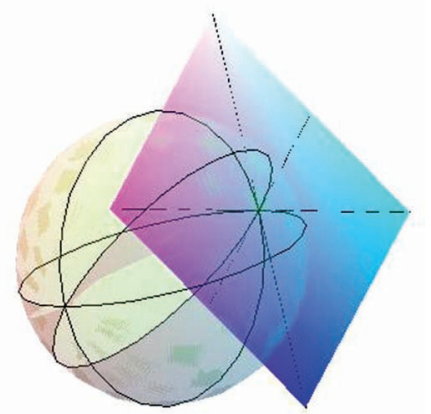

Source: author

Figure 2 Some lines in the tangent space and corresponding geodesics in the sphere

A generalization of the Euclidean space is the Minkowski space, which is a vector space equipped with a norm function $F$. Of course, $F$ may be the above norm induced by the scalar product, but there are more general norms. It is natural to require that the norm is positively homogeneous and strictly convex. Simplest examples are Randers norms, which are determined by a symmetric positively definite bilinear form $a$ and a one-form $b$ whose length with respect to $a$ is smaller than one. For any vector $v$, its norm is given by the formula

$$
F(v)=\sqrt{a(v, v)}+b(v) .
$$

The set of unit vectors with respect to a Randers norm is not the circle anymore (or the sphere, in general), but the ellipse (or ellipsoid, in general) which is not centered at the origin. Because the norm of the one-form $b$ with respect to the bilinear form $a$ is smaller than one, the origin $o$ of the vector space is inside this ellipse. For example, if $a$ is the standard bilinear form in dimension 2 with components given by the unit matrix and $b$ is the one form such that $b_{1}=0$ and $b_{2}=\frac{1}{2}$, then the ellipse of unit vectors is indicated on the picture below.

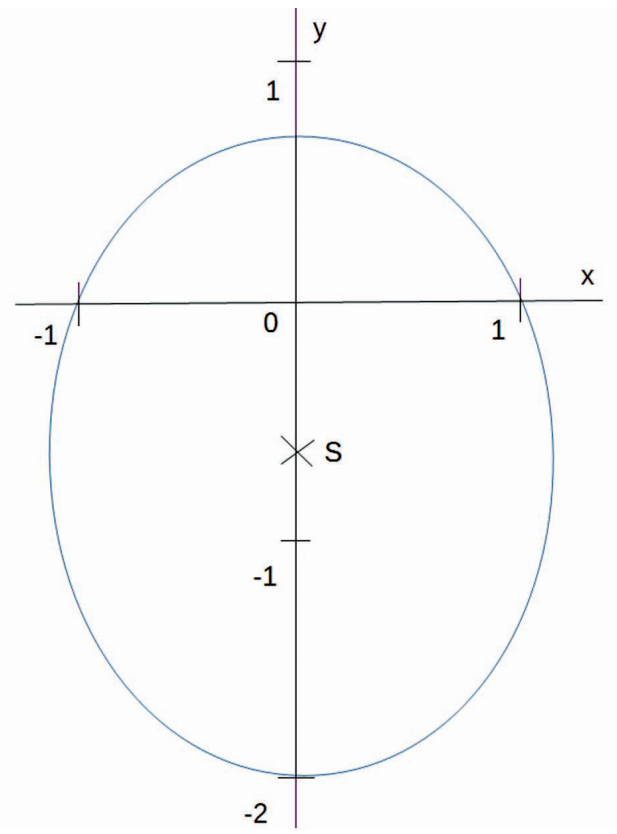

Source: author

Figure 3 Unit vectors with respect to a Randers norm

Now, a Finsler space $(M, F)$ is a smooth manifold $M$ and the Finsler function $F$, which gives a Minkowski norm on the tangent space at any point $x$ of $M$. If these Minkowski norms are Randers norms, the Finsler space $(M, F)$ is called Randers space. In the similar way as for the Riemannian manifold, in the Finsler space $(M, F)$, we naturally define the length of a curve $\sigma$ defined on the interval $(a, b)$ as the integral of norms of tangent vectors $\sigma^{\prime}(t)$ at points $\sigma(t)$ along this curve, hence

$$
L(\sigma)=\int_{a}^{b} F\left(\sigma(t), \sigma^{\prime}(t)\right) d t .
$$

However, in a Finsler space $(M, F)$, there are strong differences compared with a Riemannian manifold $(M, g)$. First, the Minkowski norm is not symmetric in general and hence $F(v) \neq F(-v)$ in general. Because of this, the length of a curve $\sigma$ travelled in one direction may be different that the length of the same trajectory travelled in the opposite direction. Also a curve may be a geodesic if we parametrize it in one direction and the same trajectory may not be a geodesic if we parametrize it in the opposite direction. But it remains true in the complete Finsler space $(M, F)$ that for any $x$ of $M$ there exist a neighbourhood $U$ of $x$ such that any $y$ of $U$ can be joined with $x$ by the unique minimizing geodesic lying in $U$. See for example the monographs [5] by D. Bao, S.S. Chern and Z. Shen or [7] by S. Deng for more details about Finsler geometry.

\section{NAVIGATION DATA AND RELATED RANDERS METRIC}

Let us now return to the real problem of the ship on the sea. In the simplest case, the surface of the sea can be considered to be the two-dimensional space $E^{2}$ equipped with the standard norm as in the Introduction. In the more general case, the sea can be modelled by the two-dimensional sphere with the natural Riemannian metric. However, in full mathematical generality, 
the sea can be any Riemannian manifold of arbitrary dimension. The possible tracks of the ship are curves on the manifold and it is natural to aim at the destination in the shortest possible time. To measure the length of particular trajectories, or the time necessary for travelling these particular trajectories, respectively, we use the Riemannian metric $h$ on $M$ and the integral above. Naturally, the curves of interest are geodesics with respect to this metric.

Now consider the same situation with the wind or with the current. This problem was originally formulated by Zermelo in [16] for the manifold $M=E^{2}$ and later it was generalized for arbitrary manifold $M$. See also the book [6] by C. Carathéodory for a modern formulation using partial differential equations and the corresponding variational problems. The direction and the strength of the wind or of the current are represented by the vector $W_{x}$ at each point of the sea. We assume that the wind is not too strong, more precisely, at any point $x$, it holds $h\left(W_{x}\right.$ $\left.W_{x}\right)<1$. We further assume that the wind is not changing in time, however, at different points it may have different strength and different direction. At any point $x$ of the manifold $M$, the wind or the current is represented by the vector from the tangent space at $x$. We shall consider just one point and its tangent space, which is the Euclidean space. The wind at this point is represented by just one vector $W$. If a ship is navigated to go in direction of a vector $v$ (representing the velocity, which has the direction and the value), the real direction of the ship is $v+W$. Hence, $v+W$ is the real movement of the ship in the unit time. The Riemannian metric $h$ (which gives the scalar product $h_{x}$ and consequently the norm at each point $x$ of $M$ ) and the vector field $W$ (which gives the vector $W_{x}$ at each point $x$ of $M$ ) are called Zermelo navigation data.

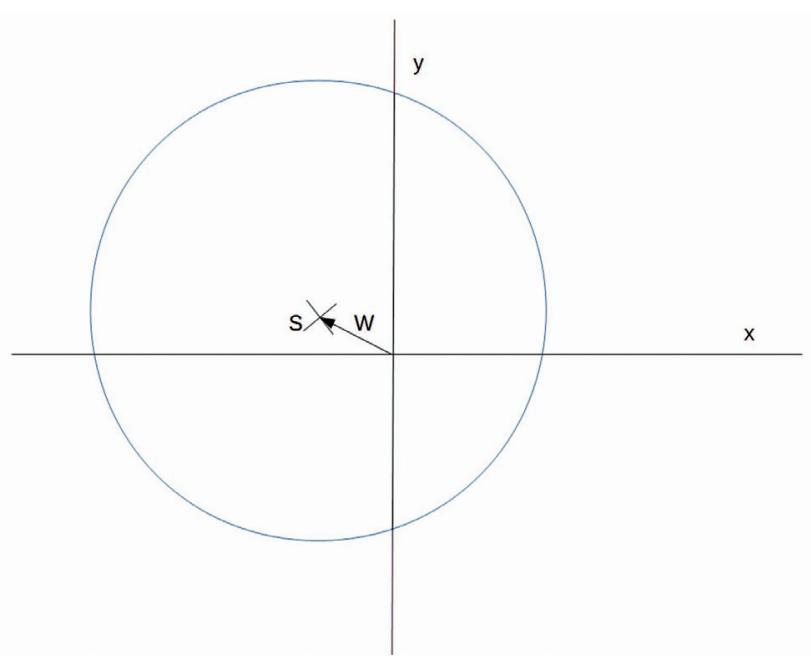

Source: author

Figure 4 Distance travelled in unit time with the influence of W

We are going to show that the navigation data (a Riemannian metric $h$ and a vector field $W$ ) determine a unique Randers metric $F$. The details can be found for example in the paper [3] by D. Bao, C. Robles and Z. Shen or the paper [13] by C. Robles. We shall work locally on a neighbourhood of a point $x$ of $M$. The components of the Riemannian metric $h$ with respect to some coordinate system are $h_{i j}$ and the components of the vector field $W$ are $W$. We further denote by $\lambda$ the function

$$
\lambda=1-|W|^{2}=1-h_{i j} W^{i} W^{j}
$$

and we denote by $W_{i}$ the components of the dual one-forms, hence $W_{i}=h_{i j} W$. Then the functions

$$
a_{i j}=\frac{h_{i j}}{\lambda}+\frac{W_{i}}{\lambda} \cdot \frac{W_{j}}{\lambda}
$$

are components of the symmetric bilinear form $a$ and functions

$$
b_{i}=-\frac{W_{i}}{\lambda}
$$

are components of the one-form $b$. Using these forms, we obtain a Randers norm $F$, by the formula

$$
F(v)=\sqrt{a(v, v)}+b(v)
$$

which holds for arbitrary vector $v$. From the assumption $h(W, W)$ $<1$, we obtain the necessary property $a(b, b)<1$. The relation of the most effective trajectories on the original Riemannian manifold $(M, h)$ under the influence of the wind or the current $W$ with geodesics of the Randers norm $F$ was investigated in several works, originally for the Riemannian manifold $M=E^{2}$ and later in full generality. It was proved by Z. Shen in the paper [14] that the most effective trajectories on $(M, h)$ under the influence of the wind or the current $W$ are geodesics of the Randers metric $F$ constructed above.

It is also interesting to consider the inverse problem, namely start with a Randers metric $F$ and construct a Riemannian metric $h$ and the wind $W$ such that geodesics of $F$ are the effective trajectories on the Riemannian manifold $(M, h)$ under the influence of $W$. To this account, denote by $a_{i j}$ and $b_{i}$ the components of the Randers metric $F$. Denote by $a^{i j}$ the inverse matrix to $a_{i j}$ and by $b_{i}$ the components of the dual forms, namely $b^{j}=a^{i k} b_{k}$ and put

Now put

$$
\varepsilon=1-a^{j k} b_{j} b_{k}
$$

and

$$
h_{i j}=\varepsilon\left(a_{i j}-b_{i} b_{j}\right)
$$

$$
W^{j}=-\frac{b^{j}}{\varepsilon}
$$

We have obtained the components of the Riemannian metric $h$ and the components of the vector field $W$. Now, the assumption $a(b, b)<1$ implies $h(W, W)<1$. It can be checked by the direct calculations that the application of the procedure above to the pair $(h, W$ ) gives back the original Randers norm $F$.

We can conclude that the formulation of the problem using the Randers norm $F$ or using the Riemannian metric $h$ and the vector field $W$ are equivalent. For more details about this relation, see for example the paper [4] by D. Bao and C. Robles.

\section{SPECIAL SITUATIONS}

In mathematics and applications, the objects with many symmetries attract much attention. In the case of Riemannian geometry, these are Riemannian manifolds with constant sectional curvature, namely the Euclidean plane, the sphere and the hyperbolic space (any of these, in arbitrary dimension). Geodesics in these spaces are easily described. In the Euclidean plane they are straight lines, in the sphere they are the main circles. In the hyperbolic space the description depends on the model. The generalization of manifolds of constant sectional curvature to Finsler geometry are Finsler spaces with constant flag curvature, see for example [4] for details.

In the paper [13], the relation of the Randers metrics which arise from the Zermelo navigation data $(M, h, W)$, where the vector field $W$ is an infinitesimal homothety were studied and 
geodesics of these Randers metrics were described. If, moreover, the Riemannian manifold $(M, g)$ has constant curvature, it was proved in [3] that the Randers metric obtained this way has constant flag curvature. Conversely, any Randers metric with constant flag curvature arise as the solution of Zermelo navigation problem on the Riemannian manifold $(M, g)$ with the influence of an infinitesimal homothety $W$. As a corollary, the trajectories of the Zermelo navigation problem in this situation are described.

Other special situations have recently been studied. For example, Zermelo navigation problem on special manifolds in dimension 3 and simulations in this situation were presented by p. Kopacz in [8]. Zermelo navigation problem on manifolds with special metrics was investigated for example in recent papers [1] and [2] by N. Aldea and P. Kopacz. It is also possible to study the problem using the calculus of variations, see for example the paper [10] by E. J. McShane. We mention also a recent paper [12] by R. Paláček and O. Krupková with the approach using the Euler-Lagrange equations and simple simulations. For other aspects of the Zermelo problem related to variational geometry see the recent paper [15] by Z. Urban and D. Krupka. The numerical approach to the Zermelo navigation problem using mathematical software was presented for example in the paper [11] by M. Mureşan. For possible generalizations of the problem, with fixed or moving obstacles, see the paper [9] by B. Li, Ch. Xu, K. L. Teo and J. Chu.

\section{CONCLUSION}

The mathematical formulation of the Zermelo navigation problem using Riemannian and Finsler geometry was introduced. Some basic ideas were illustrated and important relations were given. Some special situations with the complete solution of the problem were mentioned. The research in other special situations and other possible ways of the description of the problem were indicated. The potential for further study is huge, because the problem is too complex to be solved explicitly in full generality.

\section{REFERENCES}

[1] Aldea, N., Kopacz, P. Generalized Zermelo navigation on Hermitian manifolds under mild wind, Differ. Geom. Appl., 2017, Vol. 54, pp. 325-343. https://doi. org/10.1016/j.difgeo.2017.05.007

[2] Aldea, N., Kopacz, P. Generalized Zermelo navigation on Hermitian manifolds with a critical wind, Results in Math., 2017, Vol. 72, pp. 2165-2180. https://doi. org/10.1007/s00025-017-0757-6

[3] Bao, D., Robles, C., Shen, Z. Zermelo navigation on Riemannian manifolds, J. Differ. Geom., 2004, Vol. 66, pp. 377-435. https://doi.org/10.4310/ jdg/1098137838

[4] Bao, D., Robles, C. Ricci and flag curvatures in Finsler geometry, in: Some Perspectives of Finsler Geometry, MSRI Publications 50, Cambridge University Press, 2004.

[5] Bao, D., Chern, S.S., Shen, Z. An introduction to Riemann-Finsler geometry, Springer Science+Business Media, New York, 2000.

[6] Carathéodory, C. Calculus of Variations and Partial Differential Equations of the First Order, AMS Chelsea Publishing, 1999.

[7] Deng, S. Homogeneous Finsler spaces, Springer Science+Business Media, New York, 2012.

[8] Kopacz, P. Simulation of Zermelo Navitation on Riemannian Manifolds for $\operatorname{dim}(\operatorname{RxM})=3$, In: Navigational Problems - Marine Navigation and Safety of Sea Transportation, 2013, A Balkema Book, CRC Press, Taylor \& Francis Group Boca Raton - London - New York - Leiden, A. Weintrit, Ed., pp. 333-337.

[9] Li, B., Xu, Ch., Teo, K. L., Chu, J. Time optimal Zermelo's navigation problem with moving and fixed obstacles, Applied Mathematics and Computation, 2013, Vol. 224, No. 1, pp. 866-875. https://doi.org/10.1016/j.amc.2013.08.092

[10] McShane, E. J. A Navigation Problem in the Calculus of Variations, American Journal of Mathematics, 1937, Vol. 59, No. 2, pp. 327-334. https://doi. org/10.2307/2371415

[11] Mureşan, M. On Zermelo's navigation problem with Mathematica, Journal of Applied Functional Analysis, 2014, Vol. 9, No. 3-4, pp. 349-355.

[12] Paláček, R., Krupková, O. On the Zermelo problem in Riemannian manifolds, Balkan Journal of Geometry and Its Applications, 2012, Vol. 17, pp. 77-81.

[13] Robles, C. Geodesics in Randers spaces of constant curvature, Trans. Am. Math. Soc., 2007, Vol. 359, pp. 1633-1651. https://doi.org/10.1090/S00029947-06-04051-7

[14] Shen, Z. Finsler metrics with $\mathrm{K}=0$ and $\mathrm{S}=0$, Canadian J. Math., 2003, Vol. 55, pp. 112-132. https://doi.org/10.4153/CJM-2003-005-6

[15] Urban, Z., Krupka, D. The Zermelo conditions and higher order homogeneous functions, Publ. Math. Debrecen, 2013, Vol. 82, pp. 59-76. https://doi. org/10.5486/PMD.2013.5265

[16] Zermelo, E. Über das Navigationsproblem bei ruhender oder veränderlicher Windverteilung, Z. Angew. Math. Mech., 1931, Vol. 11, pp. 114-124. https:// doi.org/10.1002/zamm.19310110205 